Commercial Aircraft Composite Technology 
Ulf Paul Breuer

\section{Commercial Aircraft Composite Technology}

Springer 
Ulf Paul Breuer

Institut für Verbundwerkstoffe $\mathrm{GmbH}$

Kaiserslautern, Germany

ISBN 978-3-319-31917-9

ISBN 978-3-319-31918-6 (eBook)

DOI 10.1007/978-3-319-31918-6

Library of Congress Control Number: 2016939483

(C) Springer International Publishing Switzerland 2016, corrected publication May 2018

This work is subject to copyright. All rights are reserved by the Publisher, whether the whole or part of the material is concerned, specifically the rights of translation, reprinting, reuse of illustrations, recitation, broadcasting, reproduction on microfilms or in any other physical way, and transmission or information storage and retrieval, electronic adaptation, computer software, or by similar or dissimilar methodology now known or hereafter developed.

The use of general descriptive names, registered names, trademarks, service marks, etc. in this publication does not imply, even in the absence of a specific statement, that such names are exempt from the relevant protective laws and regulations and therefore free for general use.

The publisher, the authors and the editors are safe to assume that the advice and information in this book are believed to be true and accurate at the date of publication. Neither the publisher nor the authors or the editors give a warranty, express or implied, with respect to the material contained herein or for any errors or omissions that may have been made.

Printed on acid-free paper

This Springer imprint is published by Springer Nature

The registered company is Springer International Publishing AG Switzerland 


\section{Preface}

The contents of this book are based on my lectures "Commercial Aircraft Composite Technology" (Verbundwerkstoffe im Flugzeugbau) given to master's students at the Faculty of Mechanical Engineering, Technical University of Kaiserslautern, Germany, who wish to broaden their specific knowledge about composite material properties and manufacturing-optimised light weight design. The topic is very extensive and comprehensive textbooks only exist for certain areas. I have tried to focus on the central theme of societies' overall aircraft requirements to specific material requirements and to highlight the most important advantages and challenges of carbon fibre-reinforced plastics (CFRP) compared to conventional materials. During the product development process, it is fundamental to decide on "the right material at the right place" early on. It is, therefore, basic to understand the main activities and milestones of the development and certification process and the systematic defining of clear requirements. The process of material qualification - verifying material requirements-is explained in detail. All state-ofthe-art manufacturing technologies are described, including future perspectives. Especially for composites it is key to understand the interaction of the design scheme, manufacturing technology and resulting material properties. Here I have tried to highlight some key aspects of advanced CFRP design for primary load carrying airframe structures along with selected examples. I have also included a short chapter on testing as part of the certification process-and repair.

As more and more high-performance composites such as CFRP are also used in other sectors-especially automotive, manufacturing systems engineering, wind power, architecture, sports and leisure and medical engineering-the textbook can also be useful for students and engineers engaged in these areas.

The book closes with an outlook on some of the latest developments for future aircraft that we presently pursue at the Institut für Verbundwerkstoffe (IVW). This last chapter was supported by my assistant researchers Tim Krooß (PPS-PES blends, enabling a material performance close to PEEK at lower cost), Benedikt Hannemann (carbon metal fibre hybrid materials, enabling electrical conductivity 
and improved failure behaviour) and Moritz Hübler (morphing structures by shape memory alloy integration into fibre-reinforced polymer structures).

I would like to thank all my old colleagues and friends for their thorough proofreading and their precious hints for improvements to this text.

Kaiserslautern, Germany

Ulf Breuer

Spring 2016 


\section{Contents}

1 Introduction . . . . . . . . . . . . . . . . . . 1 General Airframe Material Requirements: The Value of Light Weight . . . 1 Aircraft Market Development. ..................... 3 Next Generation Aircraft Requirements . . . . . . . . . . . . . 10 Lighter Airframes: Contributors and Lever Arms . . . . . . . . . . . . . 14 History of Fibre Reinforced Airframe Structure . . . . . . . . . . . . 16

Carbon Fibre Consumption . . . . . . . . . . . . . . . . . . . . 19

End of Life.............................. 20

Questions.............................. 21

References............................ 22

2 Requirements, Development and Certification Process . . . . . . . . . 25

Development Process and Requirement Cascade . . . . . . . . . . . 25

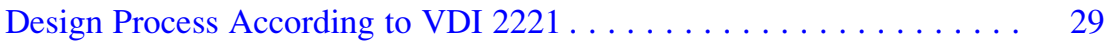

Load Cases and Stressing .................... 32

Damage Tolerance Requirements: The "No Crack Growth" Concept of CFRP ................................ 34

Certification Concept....................... 41

Questions............................. 42

Exercise: Simplified Functional Analysis . . . . . . . . . . . . . 43

References.......................... 43



Selection Criteria .......................... 45

Conventional and New Aluminium Alloys............... 47

Fatigue Crack Growth........................ 48

Fracture Toughness (Crack Resistance Curve or R-Curve) . . . . . . . 49

Fibre Metal Laminates . . . . . . . . . . . . . . . . . . . . . 50

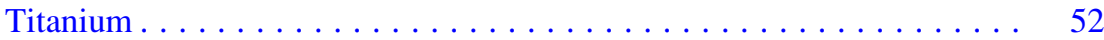




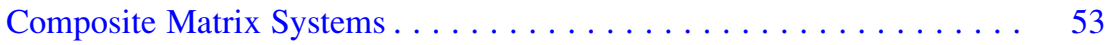

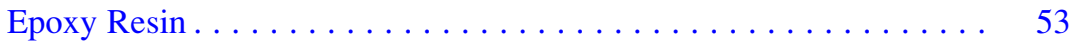

Thermoplastic Matrices . . . . . . . . . . . . . . . . . . 54

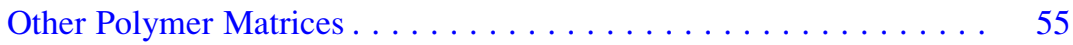

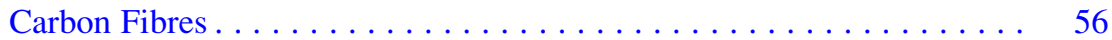

Thermoset Prepregs . . . . . . . . . . . . . . . . . . . . 60

Laminate Properties . . . . . . . . . . . . . . . . . . . . 64

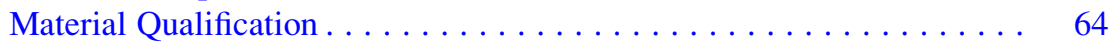

Material Qualification Process . . . . . . . . . . . . . . . 67

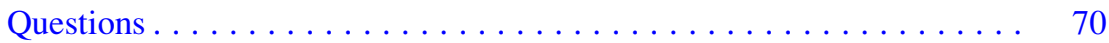

Exercise: Second Source Material Qualification . . . . . . . . . . . . 71

References ............................. 71

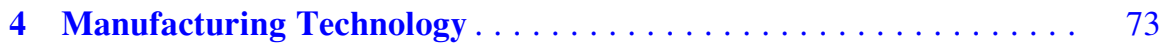

Prepreg Autoclave Technology . . . . . . . . . . . . . . . . . . . . . . . 74

Automatic Tape Laying (ATL) . . . . . . . . . . . . . . . . 76

Automatic Fibre Placement (AFP) . . . . . . . . . . . . . . 78

Pultrusion . . . . . . . . . . . . . . . . . . . . . . 79

Skin/Stringer Integration . . . . . . . . . . . . . . . . . . . . . . . . . . . . . . . . 80

Autoclave Process . . . . . . . . . . . . . . . . . . . . 81

Composite Defects . . . . . . . . . . . . . . . . . . . . . . . 84

Textile Infusion Technology . . . . . . . . . . . . . . . . . . 91

Thermoplastic Composite Technology . . . . . . . . . . . . . . . . . . . . 100

Filament Winding Technology . . . . . . . . . . . . . . . . . . . 111

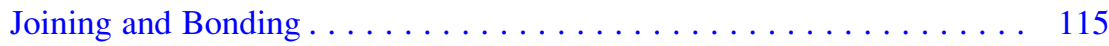

Bolted Joints . . . . . . . . . . . . . . . . . . . . . . . . 115

Adhesive Joining . . . . . . . . . . . . . . . . . . . . . . . . . . 119

Welding . . . . . . . . . . . . . . . . . . . . . 122

Manufacturing Technology Selection . . . . . . . . . . . . . . . . 123

Questions ... . . . . . . . . . . . . . . . . . . . . . . . . . . 127

Exercise: Manufacturing Technology Selection and Assessment . . . . . . 129

References. . . . . . . . . . . . . . . . . . . . . . . . . . . 129

5 Testing .............................. 133

Validation and Verification . . . . . . . . . . . . . . . . . 133

Test Pyramid . . . . . . . . . . . . . . . . . . . . . . . . . . . . 134

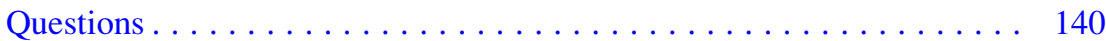

References............................ 140

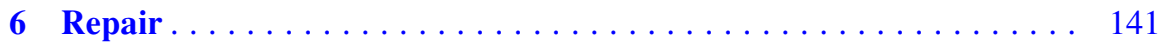

Maintenance Program . . . . . . . . . . . . . . . . . . . . . . . 141

Cosmetic and Structural Repair . . . . . . . . . . . . . . . . . . . . . . . . . 144

Bolted CFRP Repair . . . . . . . . . . . . . . . . . . . . . . . . . . . 150

Bonded CFRP Repair . . . . . . . . . . . . . . . . . . 156 
Questions ................................. 160

Exercise: Bolted CFRP Repair . . . . . . . . . . . . . . . . . . . . 160

References ............................. 161

7 Flap Design Case Study . . . . . . . . . . . . . . . . . . . 163

General Procedure, Reference Design and Targets . . . . . . . . . . . . . 163

New Design Concepts . . . . . . . . . . . . . . . . . . . . . . . . . . . . . . . . . . . . 167

Shell Sandwich Design . . . . . . . . . . . . . . . . . . . . . . . . . . . . . . . 167

Multi Spar Design in RTM . . . . . . . . . . . . . . . . . . . . . . 168

Multi Spar Design in Thermoplastic Technology . . . . . . . . . . . . 173

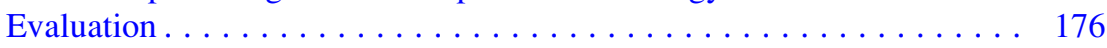



References................................. 177

8 Tailored Wing Design and Panel Case Study . . . . . . . . . . . . . . . 179

Wing Shape Control by Aeroelastic Tailoring . . . . . . . . . . . . . . . . . 179

Aeroelastic Tailoring History . . . . . . . . . . . . . . . . . . . . . . . 182

Wing Structure Stiffness Tailoring . . . . . . . . . . . . . . . . . . . . . . 184

Aileron Efficiency . . . . . . . . . . . . . . . . . . . . . . . . . . . . . 189

Flutter . . . . . . . . . . . . . . . . . . . . . . . . . . . . 191

Lift Distribution and Drag . . . . . . . . . . . . . . . . . . . 192

Strength Properties . . . . . . . . . . . . . . . . . . . . . . . . . . 193

Wing Panel Concepts . . . . . . . . . . . . . . . . . . . . . . . . . . . . . . . . . . 194

Special Manufacturing Aspects . . . . . . . . . . . . . . . . . . . 200



References............................. 210

9 New Developments . . . . . . . . . . . . . . . . . . . . . . 213

Thermoplastic Blends: Low Cost But High Performance . . . . . . . . . . 213

Polymer Blends . . . . . . . . . . . . . . . . . . . . . . . . . . . 214

Blend Morphologies . . . . . . . . . . . . . . . . . . . . . . . 215

PPS-PES Blend Developments . . . . . . . . . . . . . . . . 216

Conclusion and Outlook . . . . . . . . . . . . . . . . 219

Metal and Carbon United: Electrical Function Integration . . . . . . . . . 220

Metal Carbon Fibre Composites . . . . . . . . . . . . . . . . . . . . . . . . . 220

Material Preparation . . . . . . . . . . . . . . . . . . 223

Analytical Estimations . . . . . . . . . . . . . . . . . . . . . 224

Material Characterisation: Mechanical Properties . . . . . . . . . . . 226

Material Characterisation: Electrical Laminate Properties . . . . . . . . 227

Results and Discussion . . . . . . . . . . . . . . . . . 231

Conclusion and Outlook . . . . . . . . . . . . . . . . . 233

Shape Memory Alloy Wires: A New Approach to Morphing

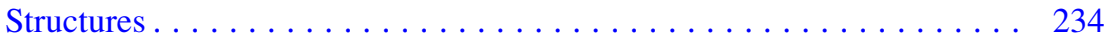

Function Integration . . . . . . . . . . . . . . . . . . 234

Solid State Actuators . . . . . . . . . . . . . . . . . . . . . 237

Shape Memory Alloys . . . . . . . . . . . . . . . . . . . . . 238 
Active Shape Memory Alloy: Fibre Reinforced Plastics Hybrid Structures . . . . . . . . . . . . . . . . . . . . . . . . . . . . . . . . . . 239

SMA FRP Manufacturing and Integration . . . . . . . . . . . . . . 239

Shape Memory Alloy Characterisation and Finite Element Model . . . 242

Potential New Applications . . . . . . . . . . . . . . . . . . . . . . . . 244

References............................ 247



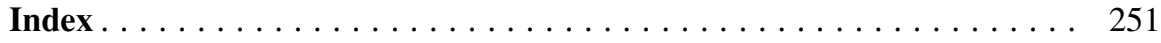




\section{Abbreviations}

$\begin{array}{ll}\text { A/B } & \text { Air brakes } \\ \text { A/C } & \text { Aircraft } \\ \text { acc. } & \text { According } \\ \text { ACD } & \text { Aircraft certification documents } \\ \text { ADL } & \text { Allowable damage limit } \\ \text { AF }_{\text {FF }} & \text { Austenite finish temperature } \\ \text { AFP } & \text { Automated fibre placement } \\ \text { Aft } & \text { Rear, back } \\ \text { AITM } & \text { Airbus industries test method } \\ \text { Al } & \text { Aluminium } \\ \text { ALI } & \text { Airworthiness limitation items } \\ \text { AMC } & \text { Acceptable means of compliance } \\ \text { AMM } & \text { Aircraft maintenance manual } \\ \text { approx. } & \text { Approximately } \\ \text { Ar } & \text { Argon } \\ \text { As } & \text { Austenite start temperature } \\ \text { ATA } & \text { Air Transport Association } \\ \text { ATL } & \text { Automated tape laying } \\ \text { Au } & \text { Aurum (gold) } \\ \text { bbl } & \text { Barrel of oil (359 l) } \\ \text { BBL } & \text { Body buttock line (fuselage width-wise position) } \\ \text { BS } & \text { Body station (fuselage lengthwise position) } \\ \text { BVID } & \text { Barely visible impact damage } \\ \text { BWL } & \text { Body water line (fuselage height-wise position) } \\ \text { C } & \text { Carbon } \\ \text { CAD } & \text { Computer-aided design } \\ \text { CAGR } & \text { Compound annual growth rate } \\ \text { CAI } & \text { Compression after impact } \\ \text { CB } & \text { Cross beam } \\ \text { CF } & \text { Carbon fibre } \\ & \end{array}$


CF/EP Carbon fibre/epoxy

CFRP Carbon fibre-reinforced plastic

CMR Certification maintenance requirements

Conf. Configuration

cont. Continued

cpt Cured ply thickness

CRC Corporate research centre

CRI Certification review items

CTE Coefficient of thermal expansion

$\mathrm{Cu} \quad$ Copper

CWB Centre wing box

DEA Dielectrical analysis

DMTA Dynamic mechanical thermal analysis

DOC Direct operating cost

DSC Differential scanning calorimetry

DSG Design service goal

EADS European Aeronautic Defense and Space Company

EAS Equivalent air speed

EASA European Aviation Safety Agency

EIS Entry into service

EKDF Environment knock-down factor

EP Epoxy

ERF Electrorheological fluid

ESG Extended service goal

ESN Electrical structural network

F/S Front spar

FAA Federal Aviation Administration

FAL Final assembly line

FAR Federal aviation regulations

FAW Fibre areal weight

FC Flight cycle

FE Finite element

FEA Finite element analysis

FEM Finite element method

FH Flight hour

FHC Filled hole compression (strength)

FHT Filled hole tension

FML Fibre metal laminate

FRP Fibre-reinforced plastic

FST Fire (or flammability) smoke toxicity

FSW Friction stir welding

FT, F/T Flap track

FTF Flap track faring

FTI Flight test installation 


$\begin{array}{ll}\text { FTIR } & \text { Fourier transform infrared spectroscopy } \\ \text { FWD } & \text { Forward } \\ \text { GF } & \text { Glass fibre } \\ \text { GFRP } & \text { Glass fibre-reinforced plastic } \\ \text { GLARE } & \text { Glass laminate aluminium-reinforced epoxy } \\ \text { GSE } & \text { Ground support equipment } \\ \text { H/S } & \text { High speed } \\ \text { H/W } & \text { Hot/wet (condition) } \\ \text { HT } & \text { High tensile strength } \\ \text { HFEC } & \text { High frequency eddy current } \\ \text { HM } & \text { High modulus } \\ \text { HMS } & \text { High modulus/high strength } \\ \text { HST } & \text { High failure strain } \\ \text { HTP } & \text { Horizontal tail plane } \\ \text { ICAO } & \text { International Civil Aviation Organization } \\ \text { IE } & \text { Impulse echo } \\ \text { ILSS } & \text { Interlaminar shear strength } \\ \text { IM } & \text { Intermediate modulus } \\ \text { IPS } & \text { Individual product specification } \\ \text { IR } & \text { Infrared } \\ \text { ISO } & \text { International Organization of Standardization } \\ \text { IVW } & \text { Institut für Verbundwerkstoffe } \\ \text { JAA } & \text { Joint aviation authorities } \\ \text { JAR } & \text { Joint aviation requirements } \\ \text { JTT } & \text { Just in time } \\ \text { L } & \text { Longitudinal } \\ \text { L/S } & \text { Low speed } \\ \text { LBW } & \text { Laser beam welding } \\ \text { LDC } & \text { Large damage tolerance } \\ \text { LEF } & \text { Load elevation factor } \\ \text { Li } & \text { Lithium } \\ \text { LL } & \text { Limit load } \\ \text { LM } & \text { Low modulus } \\ \text { LSP } & \text { Lightning strike protection } \\ \text { LT } & \text { Longitudinal transversal } \\ \text { MAC } & \text { Mean aerodynamic chord } \\ \text { MCFRP } & \text { Metal-carbon-fibre-reinforced polymer } \\ \text { MCS } & \text { Multifunctional control surfaces } \\ \text { MEK } & \text { Methyl ethyl ketone (cleaning agent) } \\ \text { MF } & \text { Metal fibre } \\ \text { Mg } & \text { Magnesium } \\ \text { Mini-TED } & \text { Master geometry } \\ \text { MLG } & \text { Main landing gear } \\ & \end{array}$




\begin{tabular}{|c|c|}
\hline $\mathrm{MoC}$ & Means of compliance \\
\hline MP & Maintenance programme \\
\hline MPD & Maintenance planning document \\
\hline MRO & Maintenance, repair and overhaul \\
\hline MS & Material specification \\
\hline MTOW & Maximum take-off weight \\
\hline MVI & Modified vacuum infusion process \\
\hline MWE & Manufacturing weight empty \\
\hline MWNT & Multi-walled nanotubes \\
\hline MZFW & Maximum zero fuel weight \\
\hline $\mathrm{NC}$ & Numerical control \\
\hline $\mathrm{NCF}$ & Non-crimp fabric \\
\hline NDT & Non-destructive testing \\
\hline NGA & Next generation aircraft \\
\hline NLG & Nose landing gear \\
\hline NRC & Non-recurring cost \\
\hline $\mathrm{OC}$ & Operating COST \\
\hline $\mathrm{OHC}$ & Open hole compression \\
\hline OHT & Open hole tension \\
\hline PAN & Polyacrylnitrile \\
\hline Pax & Passengers \\
\hline PB & Plumbum (lead) \\
\hline PEEK & Polyetheretherketon \\
\hline PES & Polyethersulfone \\
\hline PLB & Pin loaded bearing (strength) \\
\hline PLB ult. & Pin loaded bearing ultimate (strength) \\
\hline PLC & Plain loaded compression \\
\hline PLT & Plain loaded tension \\
\hline PPS & Polyphenylene sulfide \\
\hline ProHMS & Prozesskette Hochauftrieb mit multifunktionalen Steuerflächen \\
\hline PZT & Lead zirconate titanate \\
\hline QA & Quality assurance \\
\hline R curve & Residual strength curve \\
\hline $\mathrm{R} \& \mathrm{D}$ & Research and development \\
\hline $\mathrm{R} / \mathrm{S}$ & Rear spar \\
\hline $\mathrm{RC}$ & Recurring cost \\
\hline RF & Reserve factor \\
\hline RFI & Resin film infusion \\
\hline RI & Resin infusion \\
\hline RI & Repeated interval \\
\hline RPB & Rear pressure bulkhead \\
\hline RPK & Revenue passenger kilometres \\
\hline RT & Room temperature \\
\hline RTM & Resin transfer moulding \\
\hline
\end{tabular}


RTM6 Special HexFlow ${ }^{\circledR}$ Hexcel Resin (Epoxy) for resin transfer moulding

SAM Space allocation model

Sc Scandium

SEM Scanning electron microscopy

SF Safety factor

SFRP Steel fibre-reinforced plastic

SHM Structural health monitoring

Si Silicium

SMA Shape memory alloy

SRM Structural repair manual

SW Sandwich

$\mathrm{T} \quad$ Transversal

T/C Ratio air foil thickness to chord ratio

T/E Trailing edge

tbd To be determined (or: to be defined)

TC Type certification

TEM Scanning tunnelling electron microscopy

tex Unit of measure for the mass per length of rovings or yarns,

$1 \mathrm{tex}=1 \mathrm{~g} / 1000 \mathrm{~m}$

$\mathrm{Ti}$ Titanium

T-L Transversal-longitudinal

TP Thermoplastic

TS Technical specification

TTU Through transmission ultrasound

UAV Unmanned air vehicle

UD Unidirectional

UL Ultimate load

UN United Nations

US United States

UV Ultraviolet radiation

$\mathrm{V} \& \mathrm{~V} \quad$ Verification and validation

VAP $^{\circledR} \quad$ Vacuum assisted process

VDI Verein Deutscher Ingenieure

VID Visible impact damage

Vol. Volume

VSDI Visual special detailed inspection

VTP Vertical tail plane

XPS X-ray photoelectron spectroscopy

Zn Zinc 


\section{Symbols}

\begin{tabular}{ll} 
A & Area \\
$\mathrm{a}$ & Crack length \\
$\mathrm{b}_{\mathrm{F}}$ & Specific fuel burn \\
$\mathrm{c}_{\mathrm{D}}$ & Coefficient of drag \\
$\mathrm{c}_{\mathrm{L}}$ & Coefficient of lift \\
$\mathrm{d}$ & Diameter \\
$\mathrm{da} / \mathrm{dN}$ & Crack growth rate \\
$\varepsilon$ & Strain \\
$\mathrm{E}$ & Elastic modulus \\
$\mathrm{F}, \mathrm{f}$ & Force \\
$\mathrm{F}_{\mathrm{tu}}$ & Force tensile ultimate \\
$\mathrm{F}_{\mathrm{ty}}$ & Force tensile yield \\
$\mathrm{G}$ & Gravity force \\
$\mathrm{g}$ & Earth acceleration \\
$\mathrm{G}_{\mathrm{ic}}$ & Fracture toughness \\
$\mathrm{k}$ & Stress intensity factor \\
$\kappa$ & Specific electrical conductivity \\
$\mathrm{L}$ & Lift force \\
$\mathrm{M}$ & Momentum \\
$\mathrm{m}$ & Mass \\
$\mathrm{m}_{\mathrm{F}}$ & Fibre mass content \\
$\mathrm{N}, \mathrm{n}$ & Number \\
$\mathrm{n}_{\mathrm{Z}}$ & Load factor (number to multiply with g, earth acceleration) \\
$\mathrm{p}$ & Pressure \\
$\Delta \mathrm{p}$ & Delta pressure \\
$\mathrm{R}$ & Range \\
$\mathrm{R}$ & Residual strength \\
$\mathrm{S}$ & Electrical conductivity \\
$\rho$ & Density \\
$\sigma$ & Stress \\
& \\
\hline & \\
\hline
\end{tabular}




$\begin{array}{ll}\tau & \text { Shear stress } \\ \mathrm{T} & \text { Temperature } \\ \mathrm{t} & \text { Thickness } \\ \mathrm{T}_{\mathrm{g}}, \mathrm{T}_{\mathrm{G}} & \text { Glass transition temperature } \\ \mathrm{V} & \text { Volume } \\ \mathrm{v} & \text { Velocity } \\ \mathrm{v}_{\mathrm{c}} & \text { Velocity during cruise } \\ \mathrm{v}_{\mathrm{D}} & \text { Diving velocity } \\ \mathrm{v}_{\mathrm{F}} & \text { Fibre volume fraction } \\ \mathrm{v}_{\mathrm{f}} & \text { Velocity flaps } \\ \mathrm{v}_{\mathrm{fe}} & \text { Velocity flaps fully extended } \\ \mathrm{w} & \text { Width }\end{array}$

